\title{
Simulation and Research of Boiler Combustion Process Based On the Improved RBF Neural Network
}

\author{
Rong Panxiang, Sun Jianpeng, Liu Zhaoyu, Yu Lin and Dong Wenbo \\ Automation college, Harbin University of Science and Technology, \\ Harbin ,150080, China \\ Sunnyvictory008@163.com
}

\begin{abstract}
Due to the use of time, machine wear degree, coal and other reasons, the original set parameters of the boiler have been unable to meet the control requirements, therefore using a large amount of data to build a real model of the power station based on the neural network, therefore, to establish a boiler combustion optimization neural network model by using of the power plant operating data. According to the shortcomings on RBF neural networks traditional training methods with slow convergence speed, easy to fall into the local minimum. Firstly, this paper Set the model to single input and single output system as the research object, optimize neural network by the particle swarm optimization algorithm. Finally, this modeling method is expanded to the multiple input multiple output system field. Use MATLAB to establish the simulation model and the simulation research, the simulation results show that improved method for combustion boiler system efficiency has been significantly improved, combustion efficiency of the entire system reached 94\%, the accuracy of the system model was significantly better than ordinary neural network, system training error controls in less than 5\%. We can see that the improved method is feasible and effective.
\end{abstract}

Keyword: RBF neural network; radial basis function; particle swarm optimization; main steam pressure

\section{Introduction}

Artificial Neural Networks [1] is an important direction of intelligent technology development, through the data collection condition, Nonlinear system modeling method that can help people better understand the nature of things running. Neural networks are frequently used in modeling, which can help people to build up the actual work structure of relationships between various factors, such as mutual influence [2]. It with many other people in the relevant fields of the lessons learned, methods, algorithms, used in combination with studies on combustion, changing look of the boiler to the boiler working environment in a good combustion state parameters of the corresponding the ratio of these data records found, timely adjustment according to working conditions, a trend of future development.

In this paper, a power plant 200 tons / hour thermal power units for the study, using the RBF network combustion process of the boiler main steam pressure loop (single-input single-output system) modeling experiment, due to the traditional neural network modeling is not ideal, so in this paper, particle swarm optimization on RBF network to improve, continue to use the improved method for the entire boiler combustion system (multi-input multi-output system) to optimize the use of relevant data on the model for simulation and verification. 


\section{RBF Neural Network Model}

Radial basis function [3] (at any point in the space between the center and purpose of a monotonic function of the Euclidean metric) as the activation function, hence the name, the neural network weight vector selection between the vector and the threshold value from the activation function as an argument, and its structure Figure 1-1, which equation can be written as: $\|$ dist $\|$.

$$
R(\|d i s t\|)=e^{-\|d i s t\|^{2}}
$$

Between the network output and ||dist|| the relationship is reversed, |dist|| becomes large, the network output is decreased, and vice versa, if zero, then the output is 1 . In Figure 1-1, there is an argument, which is the deviation can be used to correct the speed corresponding neurons. In order to solve different problems encountered in the work, we can adopt RBF neurons [4] and other neurons portfolio accordingly.

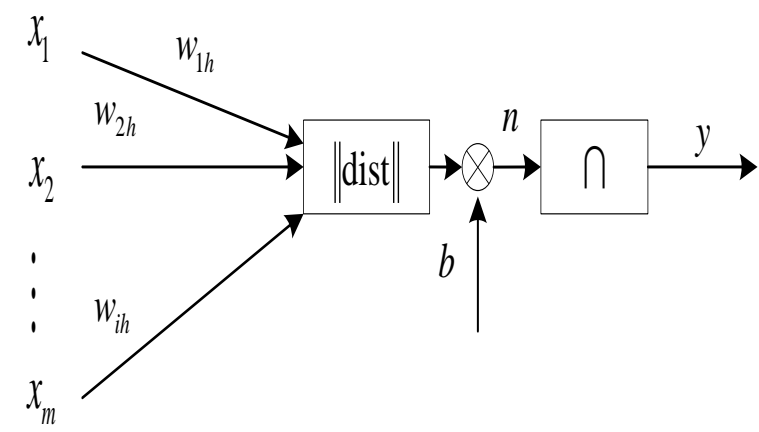

Figure 1-1. The Model of the Radial Basis Neuron

We can see from Figure 1-2 that consists of three layers of neurons combined subgroup of the basic RBF neural network. For basic RBF neural network, the first layer is used to receive data and associated data transmission, we assume that the first layer and the intermediate layer strength of the connection between them is fixed at 1 . The role of different objectives is not the same, so they choose to learn training methods are not the same. Intermediate layer using GAUSS function [5] only for network parameter correction, because it is non-linear method, so the learning rate than using a linear method for linear output layer to optimize the right a lot slower.

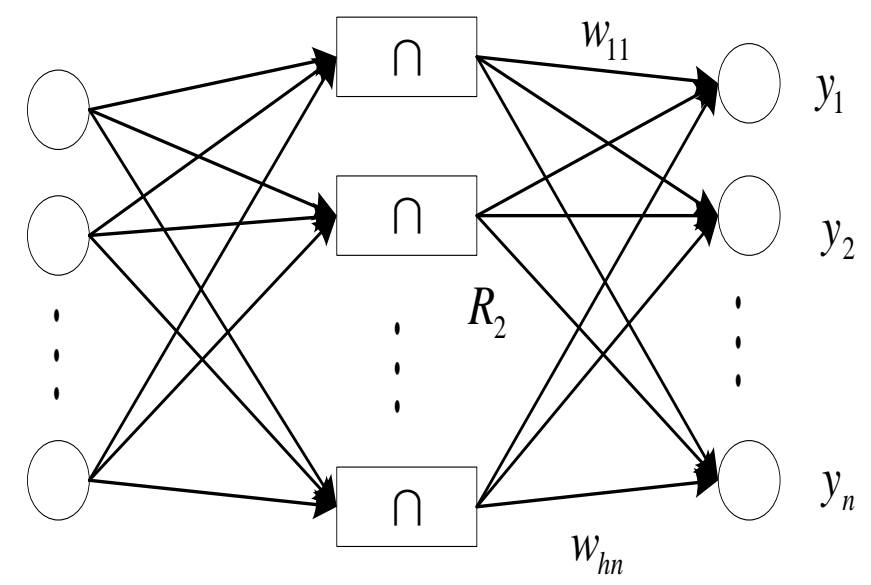

Figure 1-2. The Model of the Radial Basis Neuron Network 


\section{Particle Swarm Optimization based on RBF Neural Network Improving}

\subsection{Particle Swarm Optimization}

Particle swarm optimization [6] is an evolutionary algorithm that randomly combined with iterative methods, in order to find the best for different solutions to the problem, is built on the basis of population, the group continued in accordance with the optimal solution space particles adjustments, the there are kinds of memory function, which is different from the traditional evolutionary algorithm survival of the fittest.

Mathematical algorithm [7] is described as follows:

For example, there is an N-dimensional solution space objective, spatial memory in a population, the population consists of $\mathrm{m}$ particles, also known as population size.

We will first particle i expressed as D-dimensional position vector:

$$
z_{i}=\left(z_{i 1}, z_{i 2}, \ldots, z_{i D}\right) \quad(i=1,2, \ldots, m)
$$

Accordance with Applicable values in the known function calculates the fitness value of particles.

We use I particle speed, which is expressed as the particle displacement:

$$
v_{i}=\left(v_{i 1}, v_{i 2}, \ldots, v_{i d}, \ldots, v_{i D}\right)
$$

We use I particle to now find the best point, which is expressed as the optimal solution:

$$
p_{i}=\left(p_{i 1}, p_{i 2}, \ldots, p_{i d}, \ldots, p_{i D}\right)
$$

Now we have found the entire population of the best point:

$$
p_{g}=\left(p_{g 1}, p_{g 2}, \ldots, p_{g d}, \ldots, p_{g D}\right)
$$

According to the following formula all the particles according should be in the previous iteration to constantly adjust their speed and position:

$$
\begin{gathered}
v_{i d}^{k+1}=v_{i d}^{k}+c_{1} r_{1}\left(p_{i d}-z_{i d}^{k}\right)+c_{2} r_{2}\left(p_{g d}-z_{i d}^{k}\right) \\
z_{i d}^{k+1}=z_{i d}^{k}+v_{i d}^{k+1}
\end{gathered}
$$

In the above formula, $, i=1,2, \ldots, m, d=1,2, \ldots, D, \mathrm{k}$ is the number of iterations, particle $i$ after the $\mathrm{k}^{\text {th }}$ iteration has flight speed is expressed $v_{i d}^{k}$; that the particles after $\mathrm{k}$ iterations denoted by $z_{i d}^{k}$, the range of $r_{1}$ and $r_{2}$ is [0 1]. They can be used to maintain particle swarm diversity. In order to summarize the particles can be on their own , so that particles tend to have experienced its own best position and the whole swarm experienced the best location, We introduce two learning factors $c_{1}, c_{2}$ usually $c_{1}=c_{2}=2$, in order to avoid particles into local minimum, and to accelerate the convergence speed of particle swarm optimization, we can constantly change approach $c_{1}, c_{2}$. For different objectives, we also need to limit the range, $v_{i} \in\left[v_{\min }, v_{\max }\right]$ and $z_{i} \in\left[z_{\min }, z_{\max }\right]$. Equation (2-1) is the first iteration of the particle velocity, that it has a memory capacity of the middle term is "cognitive" section, indicating its own learning, and the final one to "society" section, on behalf of the sharing of information between the particles and mutual cooperation. By means of the formula (2-2), the particles according to the speed of the previous iteration, and in accordance with its own best experience and best practices of all particles to change the distance between the speed, and finally to the new location point. 


\subsection{Improved Clustering Algorithm}

Suppose a sample data were located within the W-dimensional space, then for all these data points, any point can be expressed as the density indicators:

$$
D_{m}=\sum_{n=1}^{a} \exp \left(-\frac{\left\|x_{m}-x_{n}\right\|^{2}}{\left(r_{1} / 2\right)^{2}}\right)
$$

In the formula, $X_{m}$ represents of the $\mathrm{m}^{\text {th }}$ sample data. The area with significantly reduced density is signified with a constant $r_{1}$. If the indicator function of this sample's point density is very large, many data dots are concentrated within the scope of this sample dot.

Through the formula below, we can get all the density indicators of data dots. From these data, choose the maximum value and record it (set as $D_{c 1}$ ) and update it as new clustering point. In this way, the density index of each data dot will change accordingly. After update, the formula can be signified as:

$$
D_{m}=D_{m}-\sum_{n=1}^{a} \exp \left(-\frac{\left\|x_{m}-x_{t s}\right\|^{2}}{\left(r_{2} / 2\right)^{2}}\right)
$$

In the formula, arising the $\mathrm{s}^{\text {th }}$ cluster center is indicated by $x_{t s}$

$$
D_{\text {max }} / D_{c 1}<r
$$

After finishing the revision of all density indicators, go on to determine the next clustering center $x_{t 2}$. If it doesn't satisfy formulate (2-5), namely, the highest density value now is much smaller than that of initial density index, repeat the above loop. If it satisfies the formula, end the clustering.

This paper explores the excellent optimizing ability of particle swarm optimization (PSO) [8] and analyzes the ability to determine the number of clustering centers with subtractive clustering algorithm, as well as the ability of K-means to keep revising the clustering centers and puts forward improvement methods. The specific process is as follows:

1). Process data and give uniform pretreatment for all the data. Find out the specific number of clustering centers (using subtractive clustering algorithm), which is also the number of center nodes in RBF network.

(1) Calculate the density of all sample dots (using formula (2-3)). Find out the maximum from these density indices. The first clustering center is the corresponding data position $x_{t 1}$ of this maximum $D_{c 1}$, renew s $=1$;

(2) Revise all the density indices (using formula (2-4)) and find out $D_{\max }$;

(3) Substitute $D_{\max }$ in formula (2-5). If it satisfies the condition, go on to the next step; if it doesn't satisfy, s $=\mathrm{s}+1$, the new clustering center is the corresponding sample position of this maximum density. Repeat the above step;

(4) Complete the operation. Finally we can get the clustering number needed $A=s$.

2) The determination of the initial particle swarm (using K-means), we generally use experimental method to determine the number of initial particles. If we specify the scale of initial particle swarm as $\mathrm{T}$, to produce $\mathrm{T}$ initial particle swarms, we need to execute K-means for T times.

(1) The number of K-means clustering centers is to determine the number of clustering 
number A and set the initial value of $t$ as 1 .

(2) By using K-means, calculate one more group of clustering centers that we need, $\left(C_{t 1}, C_{t 2}, \ldots, C_{t K}\right), t=(1,2, \ldots, T)$. In this formula, $t$ signifies clustering for the $t^{\text {th }}$ time;

(3) $t=t+1$. If $t>T$ is met, go on to the next step. If not, repeat the above step;

(4) When K-means is completed, each particle contains $T$ initial particles of $A$ clustering centers.

To generate clustering dots in accordance with the principle of arbitrariness is the feature K-means clustering. This kind of arbitrariness will cause the irregularity of clustering centers and even make some cluttering dots beyond the correct limits. While PSO uses the T types of clustering results produced in $T$ times of clustering as a method for initial particle swarm. It can reduce the serious interference or initial sample dots in k-means clustering.

\subsection{The Parameters Adjustment in Neural Network}

Neural network has a lot of parameters to be trained. There is interaction between parameters. If they are not trained properly, the final result may be affected. Major parameters include weight, width of GAUSS function and network center. The training method of network parameters [9] in this paper is PSO. The detailed steps are as follows:

1) Through the use of K-means clustering, we can determine T groups of clustering centers. Accordingly, we can get the weights between hidden layer and output layer of the $\mathrm{T}$ groups, as well as the width of GAUSS function and combine them as initial particle swarm $\left(C_{t 1}, C_{t 2}, \ldots, C_{t K}, w_{t 1}, w_{t 2}, \ldots, w_{t K}, r_{t}\right), t=(1,2, \ldots, T)$. Then PSO can be applied to adjust.

2) According to PSO, the particle swarm is constantly trained. The moving direction and speed of particles are constantly changed with the target. When it fits for the requirement (typically find out the optimizing results in accord with requirements or satisfy the expected iterations) [10], the work is over. The determination of all network parameters is completed.

\section{Modeling of the Main Steam Pressure Test based on RBF Neural Network}

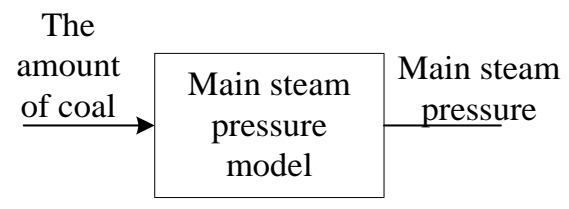

\section{Figure 3-1. The Main Steam Pressure Neuron Network}

For main steam pressure and fuel flow loop, in general, this loop mainly includes the pressure for coal and main steam. Therefore, we can use neural network, based on the actual data from scene, to set up a relational model between the two, namely the system model for single input and output.

We still adopt the 100 groups of data in a certain power plant as sample to train network and establish a RBF neural network model of single input and output between the pressure of coal and main steam. After 250 times, the network is built. Its training error curve is shown in Figure 3-1. After improvement, we get the new relevant parameters and substitute them in the formula. The relational model between main steam pressure and coal in-feed after improvement is obtained. 


$$
y=5.0451 \cdot \exp \left[-\frac{(x-146.9167)^{2}}{371.5702}\right]+6.4698 \cdot \exp \left[-\frac{(x-153.4733)^{2}}{376.01}\right]
$$

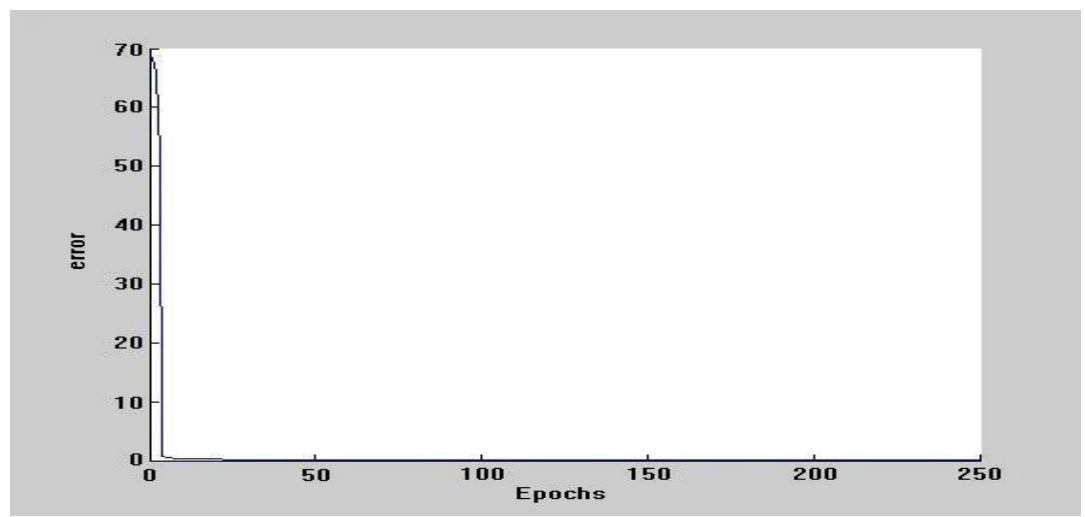

Figure 3-1. The Training Error Curve of the Main Steam Pressure

To test the feasibility of the improved method, we still use the original 100 groups of testing data, through comparing with the ordinary neural network modeling, we can see that after improvement, the error between network output and actual output is basically within 5\%, except for individual dots. It reaches the requirements for modeling parameters. Besides, the modeling effect is obviously better than that of pre-improvement model. So the established model with improved methods is suitable for actual model. The improvement methods are feasible and effective. Thus, the new model we establish with improved methods is more suitable for the relationship between main steam pressure and coal infeed.

\section{Optimization Modeling of Boiler Combusting based on Improved RBF Neural Network}

Previously, we have given modeling simulation and verification over the main steam pressure loop for the process of boiler combustion. The results show that main steam pressure system of single input and output is feasible and effective. On this basis, we simulate and verify the whole boiler combustion system with improved methods. Since actual situation of each power plant is different, the collected data are different, too. This paper aims at the production data of power plants and analyzes the relationship between boiler output and input. The coal infeed, load, induced draft fan and blower opening are selected as input, while boiler efficiency and oxygen content of flue gas are selected as output.

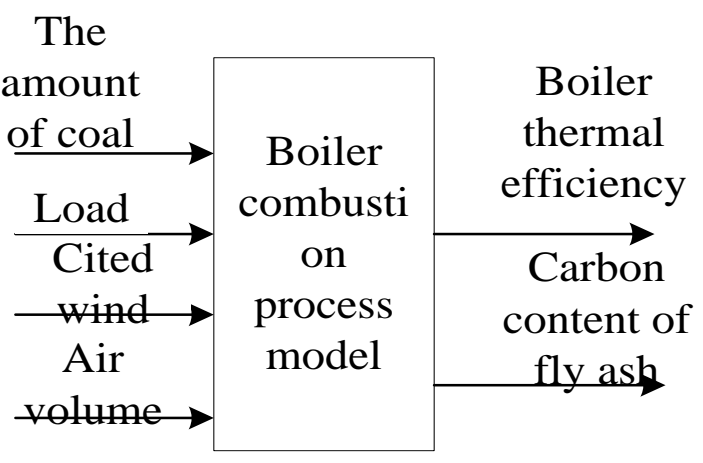

Figure 4-1. Boiler Combustion Process Model 
With coal infeed, load, air volume and output as input, the boiler heat efficiency and oxygen content in flue gas as output, a 4 input and 2 output boiler combustion process model is established. During the collection of data, we select 100 groups as the training samples of neural network modeling; As shown in Figure 4-2, we can see that after trainings for 250 times, the network training is completed. The network model for the process of boiler combustion is obtained. In this formula, $Y_{1}$ and $Y_{2}$ represent the boiler heat efficiency and carbon content in exhaust smoke. $X$ is a four dimensional vector quantity composed by four inputs. $C_{n}, \sigma_{n}$ and $w_{m n}$ represent clustering center, radius and weight respectively. The formula below is a model obtained through uniformization of data. The detailed parameters are shown in Table 4-1.

$$
\begin{aligned}
Y_{1}= & w_{11} \cdot \exp \left(-\frac{\left\|X-C_{1}\right\|^{2}}{2 \sigma_{1}^{2}}\right)+w_{12} \cdot \exp \left(-\frac{\left\|X-C_{2}\right\|^{2}}{2 \sigma_{2}^{2}}\right)+w_{13} \cdot \exp \left(-\frac{\left\|X-C_{3}\right\|^{2}}{2 \sigma_{3}^{2}}\right) \\
& +w_{14} \cdot \exp \left(-\frac{\left\|X-C_{4}\right\|^{2}}{2 \sigma_{4}^{2}}\right) \\
Y_{2}= & w_{21} \cdot \exp \left(-\frac{\left\|X-C_{1}\right\|^{2}}{2 \sigma_{1}^{2}}\right)+w_{22} \cdot \exp \left(-\frac{\left\|X-C_{2}\right\|^{2}}{2 \sigma_{2}^{2}}\right)+w_{23} \cdot \exp \left(-\frac{\left\|X-C_{3}\right\|^{2}}{2 \sigma_{3}^{2}}\right) \\
+ & w_{24} \cdot \exp \left(-\frac{\left\|X-C_{4}\right\|^{2}}{2 \sigma_{4}^{2}}\right)
\end{aligned}
$$

\begin{tabular}{|c|c|c|c|c|c|c|c|}
\hline$n$ & \multicolumn{4}{|c|}{$C_{n}$} & $\sigma_{n}$ & $w_{1 n}$ & $w_{2 n}$ \\
\hline 1 & 0.6381 & 0.6784 & 0.4951 & 0.6159 & 0.2809 & 0.7849 & 0.6251 \\
\hline 2 & 0.5101 & 0.4640 & 0.5843 & 0.5477 & 0.2563 & 1.1603 & 0.3269 \\
\hline 3 & 0.5698 & 0.7531 & 0.5927 & 0.6689 & 0.2222 & 0.9784 & 1.2057 \\
\hline 4 & 0.9331 & 0.5491 & 0.1144 & 0.3670 & 0.2417 & 0.2213 & 0.2883 \\
\hline
\end{tabular}

Table 4-1. Parameter Table

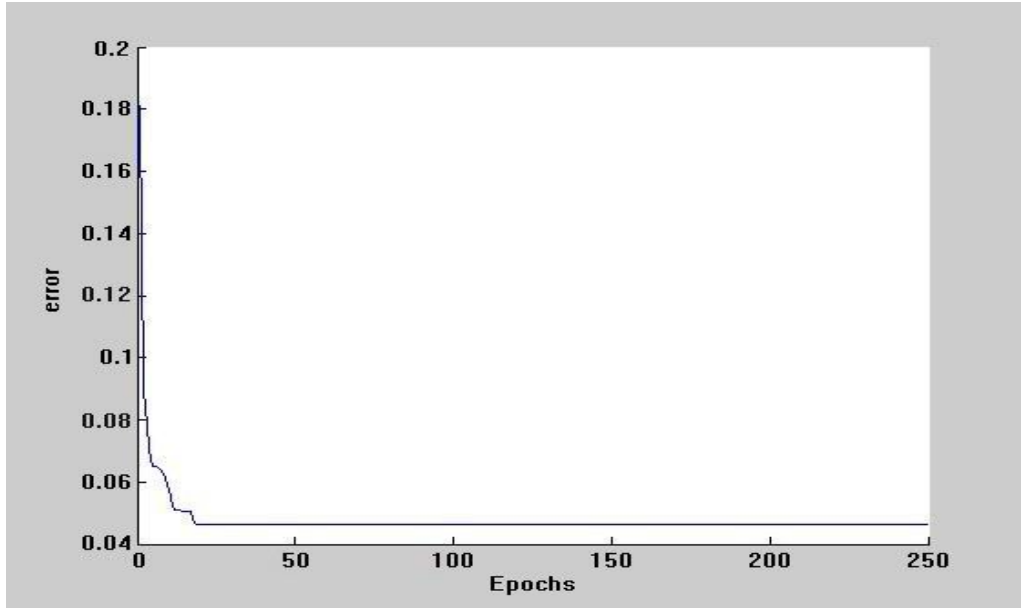

Figure 4-2. The Training Error Curve of the Combustion Processes

We can know that the improved neural network based on PSO has a high training speed for multiple-input and output system. Besides, the model is quite accurate, too. Except that some dots have big errors, there is no great difference between network output and actual output. The errors of a large majority of sample dots are controlled within $5 \%$. 
They meet the demand for actual production and explicitly reflect the working situation of boilers. Thus it is obvious that this improved method is feasible and effective for the whole combustion system.

\section{Simulation Results}

29 groups of data outside the training samples are selected as testing data. The comparison figures between improved output of heat efficiency and carbon contents and the ordinary output is obtained. The accuracy and feasibility of improved methods are verified. We can see the actual effect of improved methods more clearly.

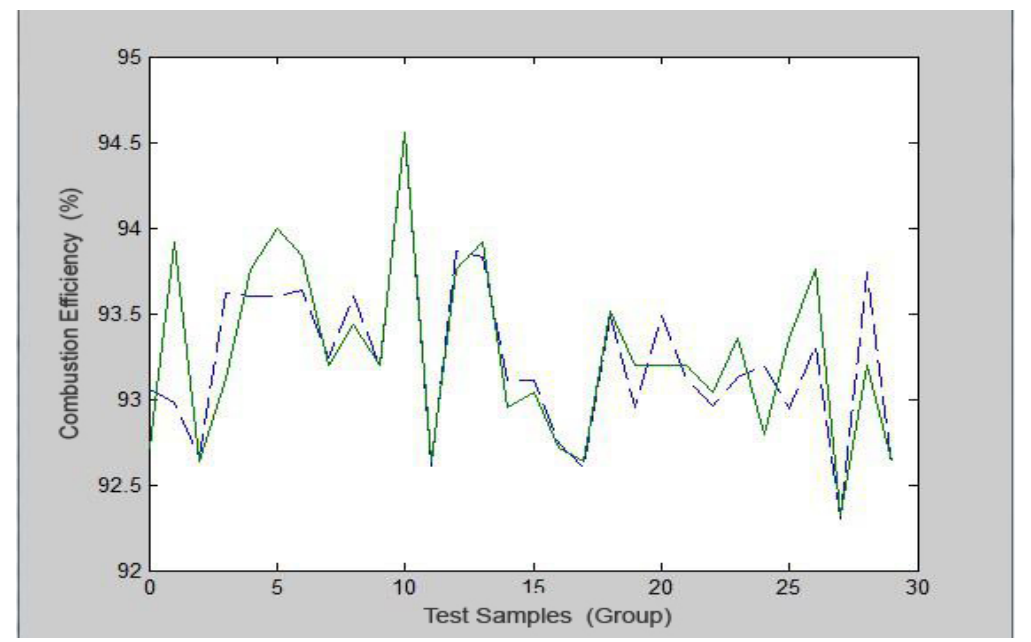

Figure 5-1. Network Output and the Actual Sample Comparison Chart of the Combustion Efficiency

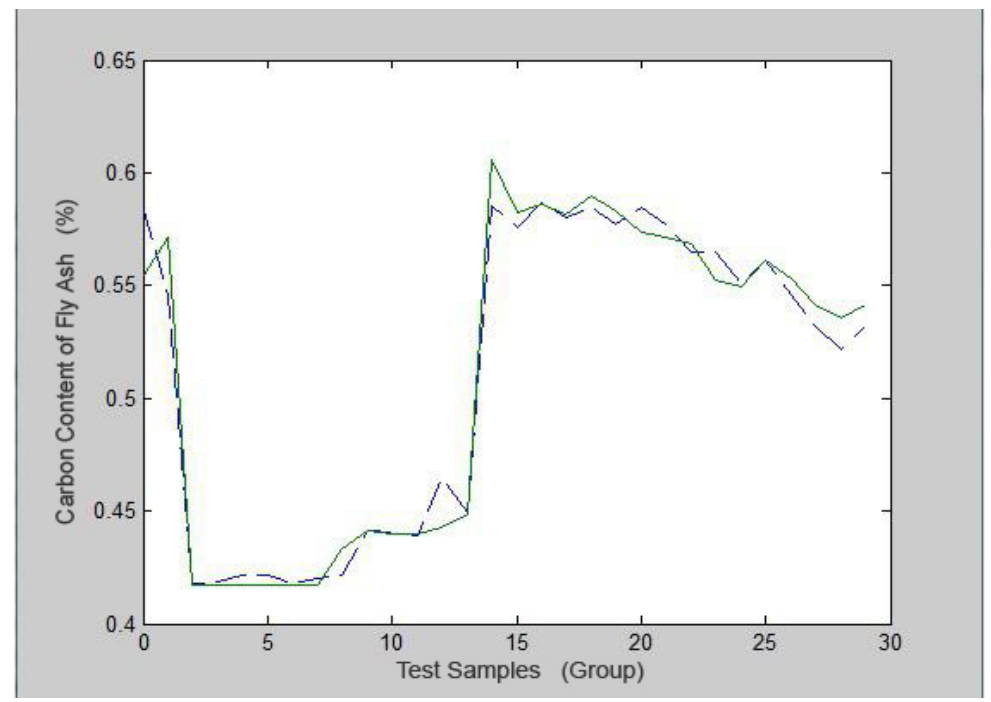

\section{Figure 5-2. Network Output and the Actual Sample Comparison Chart of the Carbon Content in Fly Ash}

Through observing Figure 5-1 and Figure 5-2, we can know that the improved neural network based on PSO has a high training speed for multiple-input and output system. Besides, the model is quite accurate, too. Except that some dots have big errors, there is no great difference between network output and actual output. The errors of a large majority of sample dots are controlled within 5\%. Moreover, the combustion efficiency of system is significantly improved, which to a large extent, reduces the production costs. 


\section{Conclusion}

This paper proposes to use neutral network for the modeling of boiler combustion system. Since ordinary neural network in the past doesn't have an ideal effect on the modeling of boiler combustion system. This paper adopts particle swarm optimization (PSO) to improve the network. The results indicate that the model set up with improved method is obviously superior to ordinary neutral network. They are actually tested in factories. The results indicate that after improvement, the combustion system is very accurate. The utilization rate of system combustion is further improved, too, thus effectively reducing the production costs.

\section{Acknowledgements}

This work was supported by Heilongjiang Provincial Department of Education Science and Technology Research Project (11551093).

\section{References}

[1] C. Lei, "Research in Boiler Combustion Optimization based on Neural Networks”, Changsha: Changsha University of Science and Technology, (2009), pp. 9-18.

[2] S. M. Thai, A. Z. S. Chong, S. J. Wilcox and J. Ward, "Neural network modeling \& control of stoker-fired boiler plant", 2007 Proceedings of the ASME International Design Engineering Technical Conferences and Computers and Information in Engineering Conference essay collection, vol. 9, (2007), pp. 575-582.

[3] Y. Along, "Eddy current sensor Non-linearity compensation method based on RBF neural network", Electrical Automation, vol. 5, (2008), pp. 66-68.

[4] C. Ming and X. Xiangdong, "Thermodynamic system fault diagnosis based on RBF neural network", Journal of Tsinghua University(Science and Technology), vol. 2, (2003), pp. 277-280.

[5] L. V. Zehua, J. Hai and Y. Pingpeng, “A Fuzzy Clustering Algorithm for Interval-Valued Data Based on Gauss Distribution Functions”, ACTAELECTEONICA SINICA, (2010), pp. 295-300.

[6] Z. Dan, H. Shengju and L. Jian, "BP Algorithm Based on Improved Particle Swarm Optimization", Computer Simulation, vol. 2, (2011), pp. 147-150.

[7] C. Shaoxin, "Research on multi-objective optimization particle swarm algorithm and its application", Dalian: Dalian University of Technology, (2007), pp. 11-35.

[8] W. Cuiru, H. Zhiqiang and Y. Hejin, "A novel RBF neural networks training method based on an improved particle swarm optimization and substrative clustering algorithm”, Journal of Computer Research and Development, vol. 42, (2005), pp. 293-298.

[9] Z. Chenjie and Y. Yongli, "Research of mapreduce based on BP neural network algorithm", Microcomputer Application, vol. 10, (2012), pp. 9-12.

[10] L. Bilian, P. Kui and S. Shaojian, "HDP-based optimizing control for boiler combustion system", Control and Instruments in Chemical industry, vol. 38, (2011), pp. 516-520. 
International Journal of $\mathrm{u}-$ and $\mathrm{e}-$ Service, Science and Technology Vol.6, No.5 (2013) 